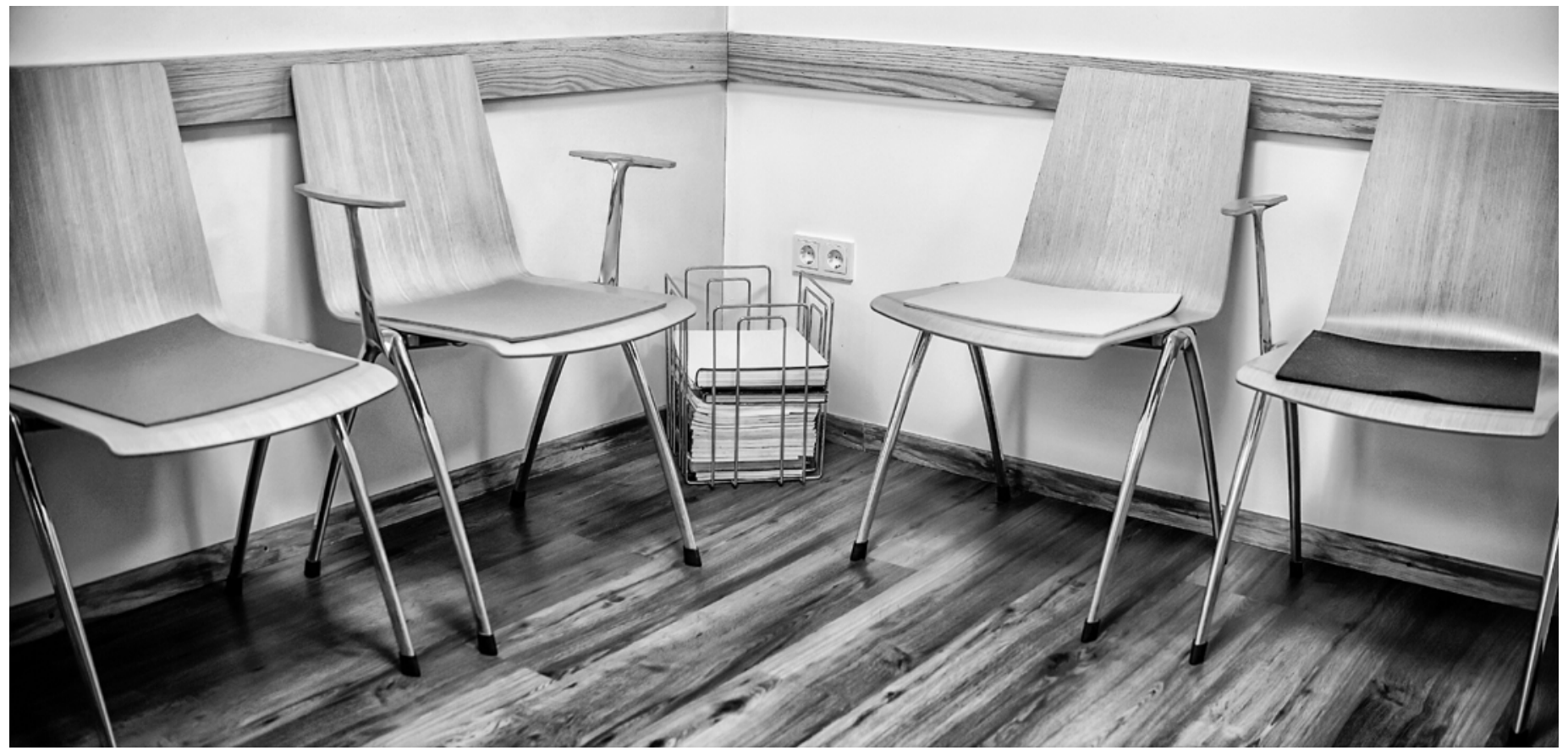

\title{
Lorsque le détenteur d'un cabinet décède
}

\section{Katharina Sigrist}

FMH Services (Fiduciaire)

A la douleur qui accompagne le décès d'un proche s'ajoute le volet administratif et les questions d'organisation, en particulier lorsque le défunt est propriétaire d'un cabinet médical. Dans ces colonnes, nous récapitulons les informations à connaître lorsqu'une entreprise individuelle se trouve dans cette situation complexe.

\section{Qui informer?}

En plus de la grande tristesse des premières heures, les proches doivent annoncer le décès dans les deux jours aux autorités suivantes:

- à l'office de l'état civil du lieu du décès, ou

- aux autorités compétentes du lieu de résidence du défunt.

Pour cela, ils présentent les documents suivants (à condition qu'ils soient disponibles):

- Acte de décès (par le médecin) ou annonce de décès

- Pièce d'identité/passeport/carte d'identité

- Permis d'établissement/permis de séjour (pour les étrangers)

- Attestation de domicile/livret de famille.
L'office de l'état civil établit ensuite l'acte de décès.

S'il est évident d'informer les proches, une information doit également être envoyée rapidement aux offices concernés par l'activité professionnelle du défunt pour faciliter les démarches administratives ultérieures.

- Fédération des médecins suisses (FMH)

- Société cantonale de médecine et organisation(s) professionnelle(s)

- Direction cantonale de la santé

- Caisse de pension

- Autres assurances personnelles

- Associations et organisations auxquelles adhérait le défunt

- Chargé du service de garde (pour autant que les gardes du défunt soient organisées par la société de 
discipline). Cela permet de réorganiser le planning avec un autre collègue.

- Fiduciaire pour que les salaires et honoraires des collaborateurs du cabinet puissent continuer d'être traités.

Il est également important qu'une personne soumise au secret médical (par ex. l'assistante médicale) communique le décès du médecin aux patients. Ces derniers doivent être informés personnellement qu'ils peuvent disposer de leur dossier médical. Pour connaître la procédure de chaque canton, il suffit de s'adresser à la société de médecine du canton concerné.

\section{Certificat d'héritier et testament}

Il est recommandé aux survivants de demander les services d'un spécialiste (par ex. avocat ou fiduciaire) pour accomplir les démarches nécessaires à l'obtention d'un certificat d'héritier ou pour régler les questions liées à la succession.

Selon les cantons, le certificat d'héritier est délivré par le conseil communal, le juge de paix ou le président du Tribunal de district. Les banques et les assurances en ont besoin pour légitimer la poursuite de la gestion du cabinet médical.

Un testament n'est pas absolument nécessaire. Les dernières volontés peuvent aussi être réglées par un pacte successoral. Si le propriétaire du cabinet a cependant rédigé un testament, celui-ci doit être transmis immédiatement aux autorités compétentes. Rappelons que le fait d'être en possession d'un testament et de ne pas le transmettre ou même de le détruire est passible de sanction. Avec l'aide de la communauté héréditaire, les autorités compétentes établissent un inventaire successoral en date du jour du décès.

\section{Engager un remplaçant pour le cabinet médical}

Dans le meilleur des cas, le remplacement en cas de décès du propriétaire du cabinet est déjà réglé et fixé par contrat. Mais dans la plupart des cas, il appartient à la communauté héréditaire d'accomplir cette tâche. Pour régler le remplacement, c'est en principe le droit cantonal qui est déterminant. La société de médecine ou la direction de la santé du canton concerné peuvent renseigner à ce sujet. Il est indispensable de fixer les modalités de remplacement dans un contrat écrit et de disposer des autorisations cantonales exigées.

L'assurance responsabilité civile du défunt devrait courir jusqu'à la fermeture du cabinet ou sa remise à un successeur. Un remplaçant qui facture des prestations avec le numéro RCC du médecin décédé et le personnel du cabinet sont ainsi toujours assurés. Après résiliation de l'assurance, les dommages imputables au défunt mais invoqués ultérieurement sont encore couverts. Cette couverture ultérieure des risques devrait correspondre aux délais de prescription légaux. Nous recommandons d'examiner ce point en détail ou de demander conseil à un spécialiste de la question.

\section{La succession}

Dans le cadre de la succession universelle, le cabinet médical est transmis aux héritiers en tant que valeur patrimoniale du propriétaire du cabinet décédé. Dès qu'ils acceptent la succession, ils en acceptent les droits mais aussi les devoirs. C'est particulièrement important pour l'obligation de conserver les dossiers médicaux pendant dix ans. Si les héritiers répudient la succession, les autorités se chargent des autres étapes.

Lorsque la succession est acceptée, la prochaine étape consiste normalement à rechercher un successeur pour le cabinet. Pour cela, il faut avoir présent à l'esprit qu'une entreprise individuelle ne peut pas être transmise en tant que telle mais qu'elle finit d'exister avec le décès de son propriétaire. Il faut donc créer une nouvelle société. Matériellement parlant, la cession partielle ou complète de l'exploitation commerciale intervient au moment du transfert des actifs et des passifs et d'une éventuelle estimation (goodwill).

Si les héritiers ne trouvent pas de successeurs, le cabinet est mis en liquidation. Dans ce cas, le conjoint survivant, les autres héritiers et les légataires - pour autant qu'ils ne continuent pas l'exploitation du cabinet médical - bénéficient d'avantages fiscaux.

\section{Contrats}

Les contrats de travail n'échoient pas avec le décès du propriétaire du cabinet. Les héritiers sont cependant autorisés à les résilier. Les préavis légaux doivent être respectés si aucun contrat de travail écrit n'existe. Dans le cas contraire, c'est le préavis inscrit dans le contrat qui s'applique. Il est cependant déconseillé de mettre trop rapidement fin aux contrats de travail du personnel du cabinet car, d'un côté, il ne faut pas sous-estimer le temps requis pour remettre ou fermer un cabinet médical et, d'autre part, il est tout à fait possible que le remplaçant ou le successeur souhaite reprendre le personnel. Cela peut avoir un effet positif sur la reprise du cabinet car le personnel connaît déjà les patients et le fonctionnement du cabinet.

La mort du propriétaire du cabinet ne met pas automatiquement un terme au bail des locaux. Les héritiers 
ont le droit de poursuivre le bail ou de le résilier en respectant les délais légaux.

Les autres contrats et conventions ne se terminent pas non plus automatiquement avec le décès du propriétaire du cabinet, à moins qu'ils aient été conclus au nom du propriétaire. Les rapports contractuels qui continuent automatiquement peuvent généralement être résiliés par les héritiers (par ex. contrat de téléphone/de fournisseurs Internet, contrat de maintenance, abonnement logiciel, revues, etc.). Mais la plupart peut être transférée au successeur du cabinet.

Enfin, comme le contrat de traitement se termine avec le décès du propriétaire du cabinet, les traitements en cours doivent être facturés. Si le cabinet ferme, les patients doivent en être informés pour qu'ils puissent récupérer leur dossier médical et chercher un autre médecin. Cette information peut se matérialiser par un courrier aux patients.

\section{Impôts}

Les héritiers sont tenus de remettre la dernière déclaration d'impôts que le défunt/testateur aurait dû rem- plir et doivent, pour cela, établir un décompte en date du décès (bilan/compte de résultats). Dans cette déclaration, ils mentionnent l'héritage ou la participation à une communauté héréditaire, même si aucune répartition n'a encore eu lieu.

\section{En conclusion}

En plus du travail de deuil lors du décès du propriétaire d'un cabinet, les survivants doivent prendre en main une multitude de détails administratifs dont, en particulier, la recherche d'un remplaçant ou d'un successeur pour le cabinet. Cette étape peut placer la communauté héréditaire face à des défis importants. Il n'existe pas de solution unique valable pour tous, chaque situation doit être considérée séparément en tenant compte de sa spécificité et des circonstances. C'est pourquoi il est recommandé de demander ponctuellement le soutien de personnes spécialisées dans les domaines concernés.

Crédit photo

(c) Irontrybex | Dreamstime.com 